\title{
Olfactory and Vomeronasal System Participation in Male Hamsters' Attraction to Female Vaginal Secretions ${ }^{1}$
}

\author{
J. BRADLEY POWERS, ROBERT B. FIELDS² AND SARAH S. WINANS \\ Neuroscience Laboratory and Department of Anatomy, University of Michigan, Ann Arbor, MI
}

(Received 5 December 1977)

\begin{abstract}
POWERS, J. B.. R. B. FIELDS AND S. S. WINANS. Olfactory and vomeronasal system participution in male hamsters' attraction to female vaginal secretions. PHYSIOL. BEHAV. 2211) 77-84, 1979.-The effects of olfactory (OLF) vomeronasal (VN), or combined deafferentations of male hamsters on their attraction to female hamster vaginal secretions (FHVS) were determined using 2 different attraction tests. In the first, FHVS was placed on one wall of a plastic test chamber, while in the second test, FHVS was rubbed onto 1 of 2 anesthetized castrate hamsters. OLF deafferentation abolished the males" attraction to FHVS in the first test but had no effect in the second. The persistence of FHVS attraction in the anesthetized castrate test depended on the VN system in that its subsequent deafferentation greatly attenuated the attraction. When the VN system was deafferented alone, FHVS attraction was significantly reduced only in males exhibiting severe mating behavior deficits. These results are interpreted to support the hypothesis that the OLF and VN systems may be preferentially responsive to volatile and non-volatile odorants, respectively. Our findings emphasize that both the OLF and VN systems participate in subserving males' attraction to FHVS and their mating with receptive females. Thus each chemosensory system may influence both sexual arousal and copulatory mechanisms.
\end{abstract}

Hamster Copulation Olfactory/vomeronasal system Sexual attraction Vaginal secretions

CHEMOSENSORY stimuli are important regulators of sexual behavior among a number of vertebrate classes $[13,29]$. The effects of these stimuli combine with those derived from other sensory and behavioral cues to initiate and coordinate reproductive interactions between male and female conspecifics. There are very few species in which one sensory modality can be singled out as crucial to reproductive activities; however, in some rodents the chemosensory modality would appear dominant. In male mice and hamsters, bilateral removal of the olfactory bulbs eliminates copulation [32]. It has been suggested that these mating behavior deficits and effects on other behaviors observed in a variety of species are due to disruption of non-sensory limbic system circuits rather than to removal of the sense of smell $[7,14]$.

Bulbectomy, as it is usually accomplished, destroys the interconnections between the olfactory bulbs and areas of the basal forebrain and brain stem $[9,11,38]$. This procedure also destroys afferents from the olfactory mucosa to the main olfactory bulb (MOB), connections of the vomeronasal organ (VNO) with the accessory olfactory bulb (AOB), the nerves of the septal olfactory organ and the nervus terminalis $[2,5]$. Although the contribution which each of these systems might make to the behavioral effects of bulbectomy in any species has not been ascertained, recent work in this laboratory has indicated that the olfactory and vomeronasal sys- tems together comprise an essential substrate in the regulation of male hamster copulation [36,42]. Although deafferentation of the olfactory system alone by intranasal infusion of zinc sulfate has no effect on mating behavior in male hamsters, combined olfactory and vomeronasal deafferentation eliminates copulation in all animals and thus mimicks the effect of bulbectomy. In addition, VNO deafferentation by itself produces a severe behavioral deficit in $20-40 \%$ of the animals but causes little or no impairment in the remaining hamsters. These behavioral observations suggest that the OLF and VN systems play complementary and interacting roles in stimulating male hamster sexual behavior.

The environmental stimuli to which the OLF and VN systems respond in promoting sexual interactions are not known, but available information suggests that components of female hamsters' vaginal secretion (FHVS) may be important for stimulating male hamsters' sexual interest. Females deposit this material by a stereotyped marking behavior [22]. Adult males sniff and lick the genital region of estrous females while engaged in copulation [6] and will spend considerable time sniffing and licking FHVS in laboratory testing situations $[8,12,17,19,25,26,27,30]$.

It has also been demonstrated that the presence of FHVS can readily alter the behavior of normal males under various social conditions. For example, the fighting which occurs

\footnotetext{
'Research supported by NSF Grant No. BMS 75-08595. We thank Anne Madsen and Kevin Alloway for valuable help in completing these experiments.

'Present address: Department of Psychology, Wayne State University, Detroit, MI 48202.
} 
between some pairs of adult male hamsters is greatly suppressed if one member of the pair is swabbed with FHVS [30], or if FHVS is simply placed on a wall of their testing chamber [31]. Additionally, FHVS applied to awake or anesthetized hamsters will stimulate other males to mount them more frequently than would occur in the absence of FHVS $[8,20,25,27,30]$.

To what extent these various responses to FHVS are mediated by either the OLF system, the VN system, or both, has not yet been established. Equally uncertain is whether FHVS might contain a variety of chemical compounds which could differentially affect these behavioral responses or chemosensory receptors. Recently, dimethyl disulfide, extracted from FHVS, has been found to stimulate active investigation by male hamsters [39]. The attractant property of dimethyl disulfide was confirmed by Macrides et al. [27], but this substance failed to elicit mounting by their males. Whether dimethyl disulfide can suppress aggressive behavior has not yet been reported.

The general strategy of the experiments reported below was to determine whether the effects of OLF and VN deafferentation on male hamster mating behavior would be paralleled by comparable changes in responsiveness to FHVS. Because there are a number of other chemical signals produced by females which could be important in stimulating the OLF/VN complex, it is by no means certain that the FHVS plays an essential role in promoting male copulation.

\section{GENERAL PROCEDURES}

Hamsters were obtained from Engle Laboratory Animals (Farmersburg, IN) weighing approximately $100 \mathrm{~g}$ upon arrival. They were housed individually in Wahmann cages, with food and water available ad lib, and exposed to a 14L:10D illumination cycle.

\section{Attraction to FHVS}

FHVS test. The males' interest in FHVS was determined by obtaining FHVS attraction scores in 2-min tests. In the first type of test, a male was placed in a clear Plexiglas test box ( $30 \mathrm{~cm} \mathrm{~W} \times 23 \mathrm{~cm} \mathrm{D} \times 23 \mathrm{~cm} \mathrm{H})$ for a 5-min adaptation period before the FHVS was introduced. Female hamsters used as sources of FHVS were ovariectomized (OVX) and implanted $\mathrm{SC}$ with silastic tubing (i.d. $=1.57 \mathrm{~mm}$; 0. d. $=3.18$ $\mathrm{mm}$ ) containing estradiol (E). Approximately $4-6 \mathrm{hr}$ prior to use they were administered $200 \mu \mathrm{g}$ progesterone $(P)$ in $0.1 \mathrm{ml}$ sesame oil, SC. This treatment reliably induces sexual receptivity, and it has been previously demonstrated that vaginal secretion obtained from OVX females treated with exogenous estrogen and progesterone is as attractive as secretion obtained from intact females in behavioral estrus [8]. FHVS collected by gently swabbing the vaginal region with a small cotton applicator was rubbed onto one wall of the test box over an area approximately $10 \mathrm{~cm}$ long and $1.5 \mathrm{~cm}$ high, located $6 \mathrm{~cm}$ from the cage floor. During this application the test male was momentarily removed from the box. When the quantity of FHVS available from 1 donor was less than normal, secretions from 1 or more additional females were utilized so that approximately equivalent quantities were applied on each test, although we did not determine the exact amount.

An FHVS attraction score was based on the behavior of the test male over a 2-min period beginning immediately after the animal was replaced in the box. The amount of time spent sniffing (nose less than $0.75 \mathrm{~cm}$ from the stimulus) or licking the FHVS was recorded, as was the time engaged in these behaviors at the opposite unswabbed wall. The number of seconds investigating (sniffing or licking) vaginal secretion was considered the FHVS attraction score. After each test the box was washed thoroughly with laboratory detergent: if used repeatedly on the same day, the side to be swabbed was alternated

FHVS- test. In 2 of the experiments to be described (Experiments 2 and 3), attraction testing was modified by altering the substrate for FHVS. In this case, vaginal secretions were applied to the genital region of one of a pair of anesthetized adult male hamsters which had been castrated at least 3 weeks previously. They were placed $25 \mathrm{~cm}$ apart. ventral side down, on the floor of a Plexiglas cage measuring $46 \mathrm{~cm} \mathrm{~W} \times 30 \mathrm{~cm} \mathrm{D} \times 30 \mathrm{~cm} \mathrm{H}$. The time spent investigating each of the two castrates (licking and sniffing within $0.75 \mathrm{~cm}$ ) was recorded during a 2 -min test. An attraction score was taken as the number of seconds investigating the castrate swabbed with FHVS. In some cases, this score was expressed as a percentage of the total time investigating the swabbed plus the unswabbed animal. This was termed a relative attraction score. We did not measure separately the time spent sniffing and licking the genital regions of the stimulus castrate as opposed to other portions of the body. However, it was our general impression that for most of the time experimental males were investigating the FHVSswabbed castrate they were sniffing and licking the genital region where FHVS had been applied.

Male mating behavior tests. Copulatory performance was assessed in Plexiglas mating chambers $(30 \mathrm{~cm} \mathrm{~W} \times 23 \mathrm{~cm} \mathrm{D}$ $\times 23 \mathrm{~cm} \mathrm{H}$ ) by adapting test males to these boxes for $5 \mathrm{~min}$ and then introducing an E-P primed OVX female hormonally treated in a fashion identical to that used for obtaining FHVS. Each stimulus female was checked with a stud male to assure that lordosis could be easily elicited before being introduced to an experimental male. Mating behavior tests were terminated when the male had achieved 2 ejaculations or after $10 \mathrm{~min}$ had elapsed, whichever occurred first. All mounts, intromissions and ejaculations were recorded. Testing occurred approximately $3 \mathrm{hr}$ after lights off but was done under normal room illumination.

Peripheral deafferentation.s. Olfactory and vomeronasal deafferentations were accomplished by intranasal zinc sulfate (ZS) infusions and surgical interruptions of the vomeronasal nerves, respectively. Both techniques as performed in this laboratory have been fully described previously $[35,42]$. For OLF Deaff, ZS was administered to etherized hamsters using a rigid 18 ga tubing outer hook which accommodated a 30 ga insert. With animals on their backs and heads lowered in an inclined position, the outer hook was passed over the hard palate until its bent end entered the nasopharyngeal meatus. The insert was then extended and attached to a $1 \mathrm{ml}$ syringe from which $0.5 \mathrm{ml}$ of a 1 -day-old $\mathrm{ZS}$ solution ( $5 \mathrm{gm} \mathrm{ZnSO} \cdot 7 \mathrm{H}_{2} \mathrm{O}$ in $95 \mathrm{ml} 0.5 \% \mathrm{NaCl}$ ) was injected into the nasal cavities. Drainage of the infused solution from the nares was facilitated by gentle suction from an aspirator during infusion and thereafter for about $10 \mathrm{sec}$. As a control procedure, $0.5 \% \mathrm{NaCl}$ was administered in an identical fashion. Hamsters were fully ambulatory approximately 2-3 min after completing the infusion procedure.

VN Deaff was accomplished by cutting the VN nerves where they pass along the medial aspect of the main olfactory bulb in their course to the accessory olfactory bulbs. Hamsters anesthetized with $75 \mathrm{mg} / \mathrm{kg}$ sodium pentobarbital were secured in a stereotaxic holder. After removing a sec- 
TABLE 1

EFFECTS OF OLFACTORY DEAFFERENTATION ON ATTRACTION TO FEMALE VAGINAL SECRETIONS IN FHVS TESTS $^{*+}$

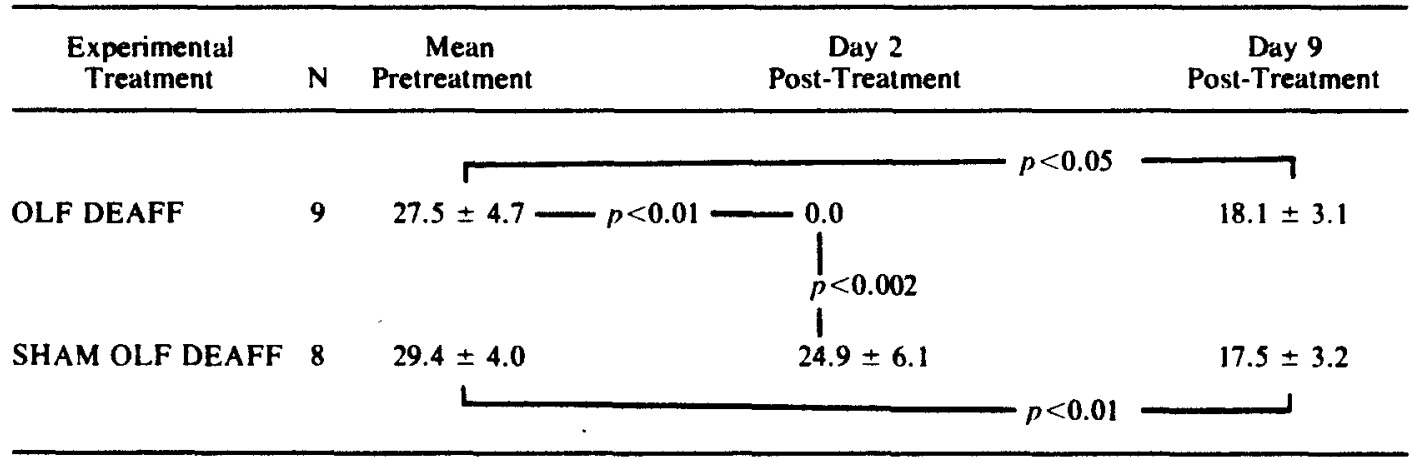

*Values are Mean secs \pm SEM.

tAll $p$ values are 2-tail.

tion of frontal bone over both OLF bulbs, the dura was punctured and open McClure Ultra Micro ophthalmic scissors were lowered over the midline sinus until the cribriform plate was encountered; after raising the scissors slightly, the blades were closed to cut the VN nerves. Sham operations were performed by duplicating this procedure except for lowering the scissors. Thus no damage was done to the MOB's or the VN nerves.

Histology. In order to determine whether our VN deafferentations were complete, all hamsters receiving this treatment were perfused under sodium pentobarbital anesthesia with $0.8 \%$ saline followed by $10 \%$ Formalin in saline. The mandible, the rostral portion of the nasal cavities, and all soft tissues were removed. The cranium was decalcified in $5 \%$ formic acid with the main and accessory olfactory bulbs in situ. When $3 \mathrm{ml}$ of a $5 \%$ sodium oxalate solution mixed with $1 \mathrm{ml}$ of the formic acid failed to produce a calcium oxalate precipitate (usually after about 4 days in acid), the decalcified cranium was transferred to $5 \%$ sodium sulfate for $14 \mathrm{hr}$, then washed in running tap water for $24 \mathrm{hr}$ before embedding. Tissues were embedded in egg yolk mixed with $12 \%$ gelatin in a ratio of $2: 1$. Forty micron frozen sections were cut in a horizontal plane through the olfactory bulbs and every 6th section was stained with cresyl violet.

When the VN nerves are completely severed bilaterally and no care is taken to avoid scarring, the central ends of the nerves degenerate, the $A O B$ glomerular layer shrinks and the glomeruli are obliterated by gliosis [42]. VN deafferentation was considered complete only if there were no remaining glomeruli in either $A O B$. If glomeruli were found, the VN cut was considered incomplete; behavioral results from these animals were either disregarded, or were considered under a "sham" category as indicated below.

Statistical evaluations. In Experiment 1, differences between experimental and control groups, and between preand post-treatment tests, were evaluated by Mann-Whitney $\mathrm{U}$ and Wilcoxon $t$-tests, respectively. In Experiments 2 and 3 $t$-tests for correlated means were used to compare pre- and post-treatment scores.

\section{EXPERIMENT 1}

We have previously shown that ZS treatment abolishes detectability of either amyl acetate or FHVS as tested by a
$\mathrm{Y}$-maze discrimination task 2 days after treatment [35]. At this time, over $90 \%$ of the olfactory epithelium is destroyed, whereas the VNO epithelium is unaffected [42]. Further, we have found that $\mathrm{ZS}$ treatment by itself has no effect on male sexual behavior but renders all animals vulnerable to the effects of VN deafferentation. That is, all hamsters with combined deafferentation of the OLF and VN systems cease copulating $[36,42]$.

In this experiment we determined whether the effects of OLF Deaff on male hamsters' attraction to FHVS would be similar to its effects on mating behavior.

\section{Method}

Seventeen males were given 2 weekly FHVS pretests and an average attraction score was calculated for each animal. Experimental and control groups had approximately equivalent mean pretest attraction scores. On the day of the final pretest, all hamsters were treated intranasally with either ZS $(n=9)$ or Saline $(n=8)$. FHVS tests were conducted again 2 and 9 days following treatment. We had previously demonstrated that 2 days after $Z S$ treatment, learned olfactory discriminations tested in a Y-maze were severely disrupted, but that by 9 days post-infusion, recovery of the discrimination had occurred in some animals [35].

\section{Results}

All hamsters were attracted to FHVS on their 2 pretests. An average of $28 \mathrm{sec}$ was spent actively investigating this substance; OLF Deaff completely eliminated FHVS attraction 2 days later. Attraction scores of OLF Deaff males were significantly lower than those of sham OLF Deaff animals $(p<0.002)$, but some interest in vaginal secretion was restored by Day 9 (Table 1). OLF Deaff males spent significantly less time investigating FHVS on both Day $2(p<0.01)$ and Day $9(p<0.05)$ than they did during pretests. Hamsters receiving the saline control treatment showed a nonsignificant decline in FHVS attraction scores on Day 2 but their Day 9 scores were significantly lower than were their pretest scores $(p<0.01)$. Thus 2 days following treatment, olfactory deafferentation abolishes interest in FHVS: the decline in Day 9 scores exhibited by both groups most likely represents a habituation phenomenon due to repeated testing. 


\section{Discussion}

ZS treatment eliminates attraction to vaginal odors in FHVS tests. This is consistent with its effects on olfactory discrimination capacities as measured by completely different techniques in our laboratory [35]. In the present experiment we tested the copulatory performance of approximately half the males in both the ZS and SAL groups 3 days after intranasal treatment; all animals achieved 2 ejaculations within 5 min. This agrees with our earlier findings that all OLF Deaff male hamsters mate normally. Thus, at a time when attraction to vaginal secretions has been eliminated in FHVS tests, males are still attracted to receptive females and engage in normal patterns of copulatory behavior with them. This suggests the paradoxical conclusion that males' attraction to FHVS, as we have measured it, plays no essential role in facilitating copulatory behavior.

The mating behavior exhibited by OLF Deaff male hamsters depends on a functional vomeronasal organ system; if the latter is additionally deafferented, mating behavior ceases and males so treated behave as if bulbectomized [42]. Thus when an OLF Deaff male interacts with a receptive female, the normal sexual behavior it exhibits depends upon sensory information received via the vomeronasal organ. Because the OLF Deaff males in this experiment had intact VN organs, one interpretation of our results is that the vomeronasal system is important for copulatory behavior but contributes little to the attraction normal males show to FHVS.

However, it could be the case that the VN system does participate in maintaining the males' interest in vaginal odors but only when the testing conditions bring the male close enough to the odor source to stimulate the VNO. Perhaps in the chain of events leading to successful copulation, the males' olfactory system functions initially by detecting volatile components of FHVS and other relevant odors at some distance. This would attract the male's interest and result in close proximity between the sexes, allowing relatively nonvolatile substances in FHVS to reach the VNO. This sequential functioning of the olfactory and vomeronasal systems might be important for maintaining sexual interest and promoting copulation.

Receptive female hamsters provide many potentially important stimuli to males in addition to vaginal odors [16]. In mating tests these cues might attract and direct the males investigatory behavior and thereby help to achieve close contact between animals. In this situation the VNO could be stimulated even if the olfactory system was non-functional, as is the case after our ZS treatment. However, in FHVS tests with no cues other than vaginal odor available, OLF Deaff males might not be able to locate the vaginal secretion because their intact vomeronasal system could respond only after close contact with FHVS had been made.

To study this idea we utilized a different FHVS attraction test in which the task of locating the FHVS, normally subserved by the olfactory system, could be accomplished by non-olfactory cues. We reasoned that if the VNO was mediating any part of the attraction to FHVS, and if these other cues were sufficient to get test hamsters close to the FHVS, then olfactory deafferentation alone should have little effect on attraction scores.

\section{EXPERIMENT 2}

The purpose of this experiment was to examine the roles of the olfactory and vomeronasal systems in mediating at traction to FHVS using tests in which vaginal secretions were swabbed onto 1 of 2 anesthetized castrate hamsters rather than onto one wall of a Plexiglas box. We assumed that the visual, tactile and perhaps other characteristics of the anesthetized hamsters would be sufficient to promote active investigation on the part of the test males, and in the process allow their vomeronasal systems to be stimulated. It has already been demonstrated that the application of FHVS to awake or anesthetized hamsters is sufficient to stimulate preferential investigation and mounting by test males $[8,20$. $25,27,30]$.

\section{Method}

Thirty male hamsters were given 2 FHVS attraction pretests on consecutive weeks. One of these was an FHVS test (same as Experiment 1) while the other was an FHVS- test (see General Method). The order in which they were presented was counterbalanced across all animals. After the second pretest an arbitrary criterion was used to select hamsters for continued use; only if the relative attraction score in the FHVS- test was $\mathbf{6 0}$ or greater was the test hamster included in the experiment. Thus for the 18 animals that met this criterion the time investigating the FHVSswabbed castrate was at least $60 \%$ of the total investigation time. This assured that these males investigated the FHVSswabbed castrate for a significantly longer duration $(p<0.001)$ than they investigated the other anesthetized castrate.

Five days after the 2 nd pretest, hamsters were administered either intranasal $Z S(n=9)$ to produce olfactory deafferentation or intranasal saline as a control procedure $(n=9)$. Animals for the experimental and control groups were chosen such that the average pretreatment attraction scores were approximately equal for both groups on the FHVStest as well as on the FHVS test. Two days after ZS or SAL treatment all animals were tested for attraction using both tests. The testing sequence was counterbalanced within both groups and approximately $1-1.5 \mathrm{hr}$ elapsed between each animal's two tests on that day.

\section{Results}

Pre- and post-treatment attraction scores are shown in Fig. 1. Experimental and control animals were well matched prior to the deafferentation procedure. The higher attraction scores on the FHVS- test indicate that various cues in addition to vaginal odors contribute to the investigation times.

The effects of OLF Deaff on FHVS attraction were critically dependent upon the particular testing situation used. Although ZS treatment virtually abolished attraction on the FHVS test $(p<0.01)$, it had essentially no effect on attraction scores when they were obtained using the FHVS- test. Animals in the sham OLF Deaff groups displayed no significant decrements in attraction scores on either test. Although not shown in Fig. 1, this profile of effects was identical to that obtained when investigation times were expressed as relative attraction scores (see General Procedures).

The fact that male hamsters can be attracted to FHVS following OLF Deaff is consistent with the hypothesis that the vomeronasal system is capable of mediating this behavior if the testing conditions are appropriate. To evaluate this more directly, we determined whether attraction to vaginal 


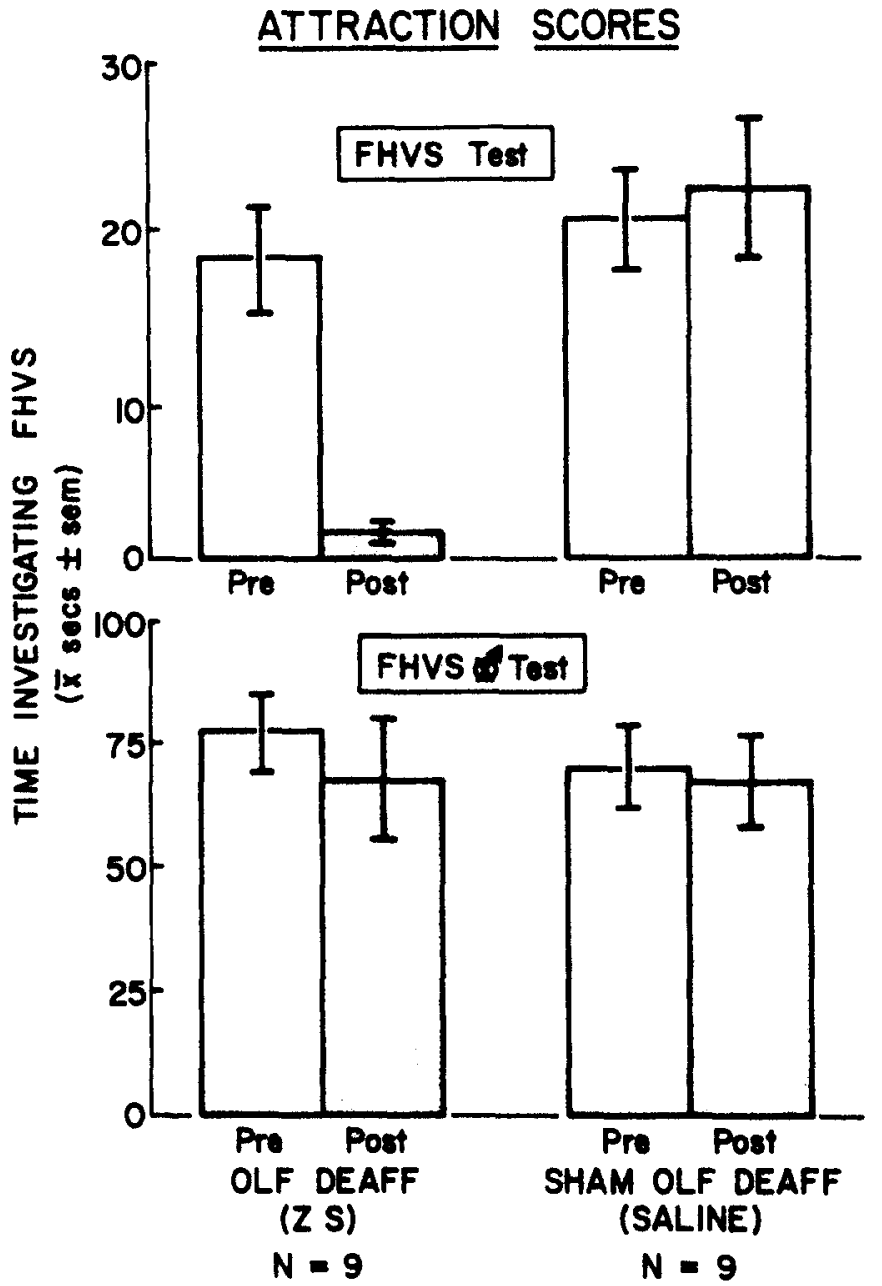

FIG. 1. Attraction to female vaginal secretions measured by 2 different tests in the same animals before and after olfactory deafferentation or sham treatments. OLF Deaff significantly reduced attraction scores $(p<0.01)$ in the FHVS test, but not in the FHVS- test.

secretions in the FHVS- test would persist following combined VN and OLF Deaff.

We chose the 12 hamsters which had displayed the highest attraction scores following their previous experimental treatments. Animals having received ZS or SAL were evenly distributed between VN Deaff $(n=6)$ and Sham VN Deaff $(n=6)$ groups. Five days after vomeronasal surgery all animals were given intranasal ZS, and 2 days later an FHVSattraction test. Thus one group was retested after combined vomeronasal and olfactory deafferentation, while the other was tested after olfactory deafferentation alone.

The effects of these manipulations were evaluated with respect to pretreatment attraction scores taken from tests which occurred 9 days prior to surgery (Fig. 2). Although FHVS investigation times were reduced following both treatments, this effect was significant only in hamsters with both the vomeronasal and olfactory systems deafferented $(p<0.02)$. A significant reduction in FHVS attraction in this group was also observed when relative attraction scores (see General Procedures) were used $(p<0.01)$. Interestingly, the one animal whose results were excluded from analysis because its vomeronasal deafferentation was incomplete, was

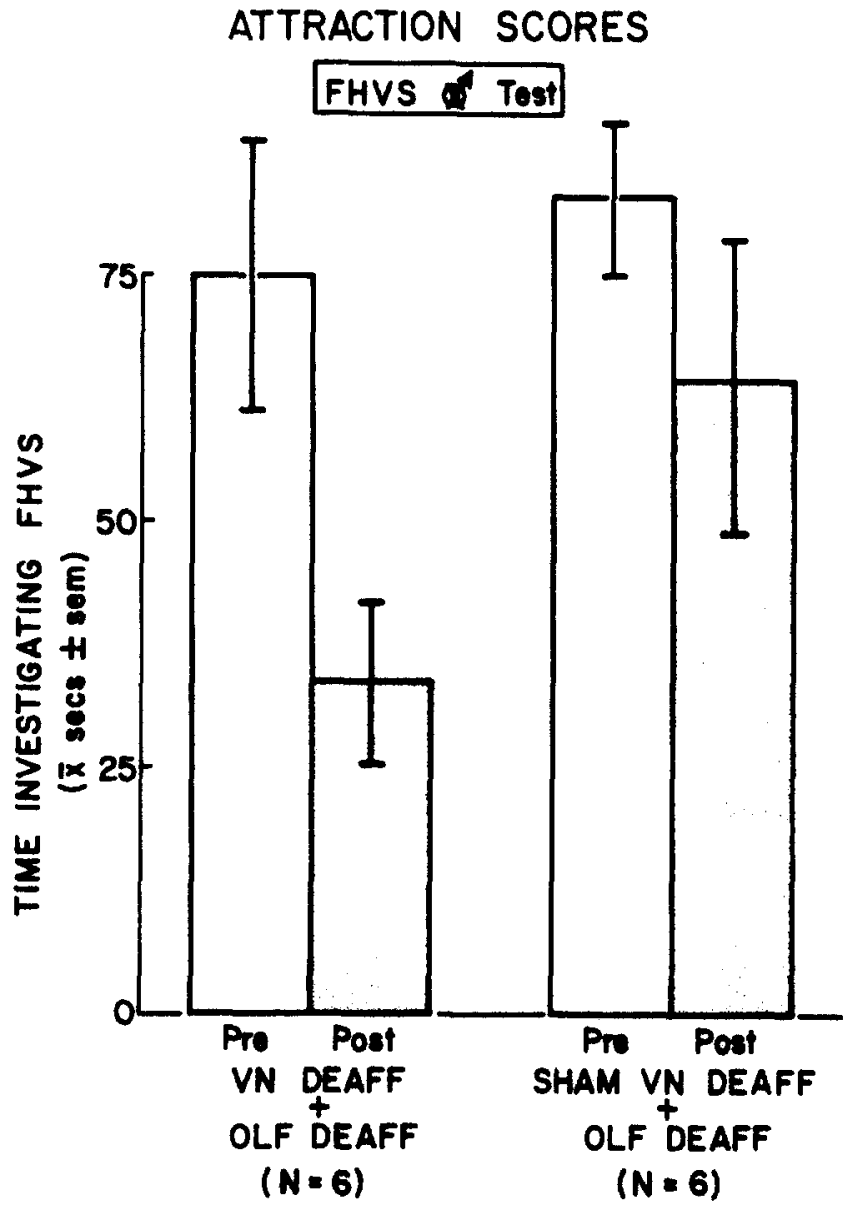

FIG. 2. Combined vomeronasal and olfactory deafferentation significantly reduced attraction scores in the FHVS- $x$ test $(p<0.02)$.

the only animal in the VN plus OLF Deaff group whose post-treatment relative attraction score $(81 \%)$ was greater than $50 \%$.

\section{Discussion}

These results confirm that interest in FHVS, as indicated by persistent sniffing and licking, is subserved by the vomeronasal system when the olfactory system has been deafferented (Fig. 1). Under these conditions, the vomeronasal organ must transduce critical information because when it also is deafferented, interest in FHVS is significantly attenuated (Fig. 2). The time these males with combined deafferentations do spend investigating the anesthetized castrates is presumably due to stimuli affecting other sensory systems.

\section{EXPERIMENT 3}

Our findings do not establish to what extent the olfactory and vomeronasal systems individually respond to FHVS when both sensory systems are intact. To gain additional insight into this problem we observed the effects of VN deafferentation alone using the FHVS- test. If the VN system normally participates in mediating male hamster's attraction to FHVS, and if FHVS plays any role in promoting copulation, a decline in attraction might be expected to occur most 
TABLE 2

EFFECTS OF VOMERONASAL DEAFFERENTATION ON ATTRACTION TO FEMALE VAGINAL SECRETIONS IN FHVS- $x$ TESTS*

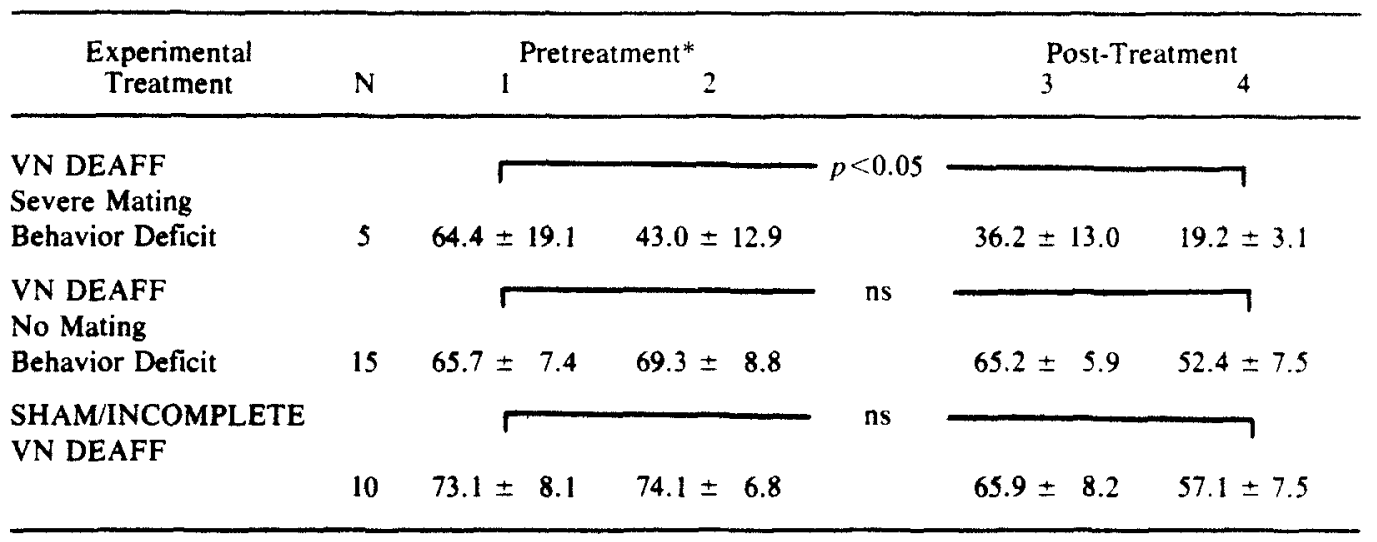

*Values are Mean secs \pm SEM on 4 successive weekly tests.

noticeably in the males exhibiting mating deficits after VN deafferentation.

\section{Method}

FHVS attraction was initially assessed in 30 hamsters over 2 successive weeks using the FHVS- test. Standard mating tests were also given once each week 2 days following the attraction tests. All males except 3 ejaculated twice on both mating pretests. These 3 showed 2 ejaculations on only 1 of the 2 pretests. No criterion was imposed with respect to FHVS attraction scores so that variability in responsiveness would not be truncated. The day after the second mating pretest all hamsters received surgical treatments which resulted in either (a) complete vomeronasal deafferentation $(n=20)$, (b) incomplete VN deafferentation $(n=5)$, or (c) a sham operation $(n=5)$. Animals in the last 2 categories were combined to form a single control group. The effects of VN surgery were evaluated over the following 4 weeks. FHVS- tests and mating tests continued for the first 2 postoperative weeks on the same schedule as was followed during the pretest. On the $3 \mathrm{rd}$ and 4 th post-operative weeks only mating behavior tests were given.

Copulatory impairments were considered present if hamsters failed to ejaculate on two or more of their four postoperative tests. This criterion is somewhat less restrictive than the one we have used in earlier publications to categorize animals as displaying severe mating behavior deficits.

\section{Results}

Five of the 20 animals with complete VN deafferentations showed noticeable mating impairments. The actual severity ranged from no mating at all on any of the post tests $(2$ animals) to ejaculatory behavior on only half of the tests ( 2 animals). The 5 males which showed these deficits postoperatively did not include the 3 that had failed to mate on one of the pretests. Fifteen VN deafferented males mated normally; 11 of these hamsters ejaculated twice on all 4 postoperative tests. This was true on 3 of the 4 tests for the remaining animals in this group. This dichotomous effect of VN deafferentation on mating behavior is comparable to what we have observed during earlier studies. No mating behavior deficits were noted in 9 of the 10 sham/incompletely deafferented hamsters; 1 failed to ejaculate on 2 of its postoperative tests.

Male hamsters' attraction to vaginal odors varied considerably over the pre- and postoperative tests. VN Deaff affected investigation of FHVS only in males which showed copulatory deficits following surgery (Table 2). This was reflected in a significant difference between their mean preand post-treatment attraction scores $(\phi<0.05)$, as well as be tween their scores on Week 1 and on Week $4(p<0.05)$. Control hamsters, and the VN Deaff hamsters which continued to mate normally after surgery did not exhibit significant decrements in time investigating FHVS.

\section{Discussion}

Our findings suggest that FHVS plays an important role in stimulating male hamster copulatory behavior; they further indicate that both the olfactory and the vomeronasal systems can respond to odorants within the female's vaginal secretions.

The results of Experiments 1 and 2 clearly demonstrate that the effects of OLF Deaff on FHVS attraction depend critically on the testing situation employed. When FHVS alone is placed on the wall of a Plexiglas test cage (FHVS test) OLF Deaff abolishes attraction to this substance $48 \mathrm{hr}$ after zinc sulfate is applied to the nasal cavities (Experiment 1). This implies that the vomeronasal system, which remains intact after our zinc sulfate procedure, either does not detect FHVS in the quantities we used, or does not mediate an investigatory response to this substance, even over the relatively short distances involved in our testing boxes. This finding is consistent with the fact that OLF Deaff also eliminates the detection of FHVS when its odor is blown from an enclosed flask through a Y-maze [35].

In Experiment 2 we showed, using an altered testing paradigm, that the vomeronasal system could subserve an investigatory response to FHVS. The time spent sniffing and licking the region of an anesthetized castrate where FHVS had been applied was not significantly reduced by OLF Deaff (Fig. 1). Thus the persistent investigation of vaginal secretory material can be displayed equally well when only the VN system is capable of responding. We hypothesize 
that under these. conditions the vomeronasal system responds because non-olfactory stimuli associated with the anesthetized castrate suffice to bring the test males into direct contact with FHVS and that this may be an important requirement for VNO activation. The involvement of the vomeronasal system in maintaining interest in vaginal secretion was further substantiated by the fact that when both the VN and OLF systems were deafferented, the time spent sniffing and licking the FHVS was significantly reduced (Fig. 2). In Experiment 3 VN Deaff alone produced attraction deficits only in those animals which also showed a concomitant impairment in their overt copulation with receptive females. Interestingly, these animals showed some decline in FHVS investigation times on Test 2 , before VN deafferentation was performed. We have suggested elsewhere [42] that males whose copulatory behavior is affected by VN deafferentation may be less vigorous maters, and in some way less easily aroused by relevant sexual stimuli than are hamsters whose mating remains normal after this procedure. Perhaps in the former animals this is reflected in the decline of their pretest attraction scores. However, it is true that all 5 of the animals showing impaired sexual behavior after VN deafferentation exhibited 2 ejaculations on each of their 2 mating pretests. In contrast, 3 of the 15 males unaffected by the deafferentation failed to mate on one of their 2 pretests. Another point to be noted concerning the decline in attraction scores of the VN deafferented males with copulatory deficits is that this decline was significant only on the second post-treatment test. This may relate to the fact that for many VN deafferented males, copulatory behavior can be quite normal on the first postoperative test, yet be entirely absent on all subsequent tests.

Based on the findings presented here and the results from our earlier studies $[35,36,42]$ we conclude that in all male hamsters the vomeronasal system, functioning in the absence of olfactory input, can subserve both persistent investigation of female vaginal secretions and overt copulatory behavior. In contrast, olfactory stimulation in the absence of vomeronasal input can mediate these 2 behavioral functions in only a subpopulation of animals. In those males in which the olfactory system is incapable of subserving these functions, we do not know whether the attenuated investigation of FHVS and the copulatory behavior deficits are causally related. Whether a proportion of $\mathrm{VN}$ deafferented males fail to mate because their attraction to and investigation of FHVS (and possibly other odors) is impaired, or whether their attraction and copulatory deficits are simply correlated, cannot be determined at present. Although some evidence suggests that male hamsters' attraction to female chemosensory stimuli can be experimentally dissociated from the ability of these stimuli to initiate mounting behavior $[8,10,27]$, hypotheses to explain the neural bases of these 2 functions must take into account the evidence that with appropriate testing procedures both the olfactory and the vomeronasal systems can participate in each.

It has long been recognized that the morphology of the VNO in mammalian species precludes easy access of odorant molecules to its receptor cells [33]. Early investigators speculated that modulation of the size of the vascular plexus surrounding the lumen of the VNO by autonomic nervous system activity could pump liquids into and out of the organ through the microscopic duct that is the organ's only access [18]. Estes [15] acknowledged that this might be true for some species, but emphasized the role of the flehmen response in providing a mechanism for odorant molecules in air to be introduced into the VNO. He proposed a model appropriate for species in which the VNO duct opens into the nasopalatine canal. This is not the case in hamsters, however, in which the VNO communicates directly only with the nasal cavities and in which the nasopalatine canal enters the nasal cavity posterior to the closed caudal end of the organ [1]. Recently, Meredith [28] has provided compelling evidence that the pumping mechanism noted above can be activated by autonomic stimulation in hamsters and can facilitate entry of odorants dissolved in liquid secretion into the VNO. Whether the same odorant molecules stimulate the olfactory and the VNO receptor cells in hamsters is not known. But it is a reasonable possibility that molecules of low volatility may not reach the olfactory receptors in sufficiently high concentration for effective stimulation. These molecules might, however, reach the VNO receptors if dissolved in liquid and directly applied to the external nares, as would occur during the male hamster's sniffing and licking of the female's ano-genital area.

Our work was the first to demonstrate conclusively the relevance of VNO activity in subserving reproductive activities in a mammalian species $[36,42]$. We have indicated elsewhere that indirect evidence suggests a comparable role for the VNO in male rats [42]. If it is true that the vomeronasal organ constitutes a chemosensory system capable of responding to non-volatile or low volatility odorants, then a number of observations on different mammalian species might relate to involvement of this organ in a variety of behavioral responses. The literature suggesting a relation between the flehmen response and stimulation of the VNO has been reviewed $[15,41]$. In more recent experimental work, comparable behaviors have been described which are exhibited in response to conspecific odors and which may function to optimize stimulus transport to the VNO: examples are the "gaping" response in cats [24], flehmen in rabbits [4] and "head-bobbing" in guinea pigs [3]. Nyby et al. [34] have studied the elicitation of ultrasonic vocalizations in male mice by female odors. Although effective odors could be obtained from a number of different body locations, a characteristic of each was that males responded to them only after a period of intense investigation at close range. It is possible, then, that stimulation of the VNO by relatively non-volatile odors is required for eliciting the male's ultrasonic calls. Although none of these observations provides direct proof of VNO involvement, they suggest that future investigations may reveal an important role of the vomeronasal system in regulating the social behavior of several vertebrate species.

In conclusion, we propose that at least 2 requirements must be met in order that the VN system in male hamsters be activated by conspecific odors. One is that the animal be in close proximity to the odorant; the ability to lick the substance may be a component of this requirement. Secondly, sufficient neural activity in the autonomic nervous system must be achieved to optimize stimulus access to vomeronasal organ receptors; this may in some cases be accomplished via OLF system responsiveness to volatile odorants. We hypothesize that when these requirements are met, the vomeronasal system in male hamsters can subserve a persistent investigation of female odors and can participate in mechanisms which initiate copulatory behavior with receptive females. The way in which the vomeronasal and olfactory systems interact and the central nervous system pathways by which their combined effect modulates reproductive activities remains to be elucidated. 


\section{REFERENCES}

1. Adams, D. R. and L. Z. McFarland. Morphology of the nasal fossae and associated structures of the hamster (Mesocricetus' auratus). J. Morph. 137: 161-180, 1972.

2. Alberts, J. R. Producing and interpreting experimental olfactory deficits. Phvsiol. Beha'. 12: 657-670, 1974

3. Beauchamp, G. K., J. G. Magnus, N. T. Shmunes and T. Durham. Effects of olfactory bulbectomy on social behavior of male guinea pigs (Cavia porcellus). J. comp. physiol. Psychol. 91: 336-346, 1977

4. Black-Cleworth, P. and G. Verberne. Scent-marking, dominance and flehmen behavior in domestic rabbits in an artificial laboratory territory. Chem. Senses Flav. 1: 465-494, 1975.

5. Bojsen-Moller, F. Demonstration of terminalis, olfactory, trigeminal and perivascular nerves in the rat nasal septum. $J$. comp. Neurol. 159: 245-256, 1975.

6. Bunnell, B. N., B. D. Bolands and D. A. Dewsbury. Copulatory behavior of golden hamsters (Mesocricelus auratus). Behaviour 61: 180-206, 1977.

7. Cain, D. P. The role of the olfactory bulb in limbic mechanisms. Psychol. Bull. 81: 654-671, 1974.

8. Darby, E. M., M. Devor and S. L. Chorover. A presumptive sex pheromone in the hamster: Some behavioral effects. $J$. comp. physiol. Psychol. 88: 496-502, 1975.

9. DeOlmos, J., H. Hardy and L. Heimer. The afferent connections of the main and accessory olfactory bulb formations in the rat: An experimental HRP study. J. comp. Netrol. 181: 213244, 1978.

10. Devor, $M$. Components of mating dissociated by lateral olfactory tract transection in male hamsters. Brain Res. 64: 437-441, 1973.

11. Devor, M. Fiber trajectories of olfactory bulb efferents in the hamster. J. comp. Neurol. 166: 31-48, 1976.

12. Devor, M. and M. R. Murphy. The effect of peripheral olfactory blockade on the social behavior of the male golden hamster. Behav. Biol. 9: 31-42, 1973.

13. Doty, R. L. (Editor) Mammalian Olfaction. Reproductive Processes, and Behavior. New York: Academic Press, 1976.

14. Edwards, D. A. Non-sensory involvement of the olfactory bulbs in the mediation of social behaviors. Behal. Biol. 11: 287-302, 1974

15. Estes, R. D. The role of the vomeronasal organ in mammalian reproduction. Mammalia 36: 315-341, 1972.

16. Floody, O. R., D. W. Pfaff and C. D. Lewis. Communication among hamsters by high-frequency acoustic signals. II. Determinants of calling by females and males. $J$. comp. physiol. Prychol. 91: 807-819, 1977.

17. Gregory, E., K. Engel and D. Pfaff. Male hamster preference for odors of female hamster vaginal discharges: Studies of experiential and hormonal determinants. $J$. comp. physiol. Prychol. 89: 442-446, 1975.

18. Hamlin, H. E. Working mechanisms for the liquid and gaseous intake and output of the Jacobson's organ. Am. J. Physiol. 91: 201-205, 1929.

19. Johnston, R. E. Sexual attraction function of golden hamster vaginal secretion. Behav, Biol. 12: 111-117, 1974.

20. Johnston, R. E. Sexual excitation function of hamster vaginal secretion. Anim. Learn. Behav. 3: 161-166, 1975.

21. Johnston, R. E. Sex pheromones in golden hamsters. In: Chemical Siunuls in Vertebrates, edited by D. Muller-Schwarze and M. M. Mozell. New York: Plenum Press, 1976, pp. 225-249.

22. Johnston, R. E. The causation of two scent-marking behaviour patterns in female hamsters (Mesocricefus auratus). Anim. Behul. 25: 317-327. 1977
23. Johnston, R. E. and N. A. Lee. Persistence of the odor deposited by two functionally distinct scent marking behaviors of golden hamsters. Behar. Biol. 16: 199-210, 1976.

24. Kolb, B. and A. J. Nonneman. The development of social re sponsiveness in kittens. Anim. Behar. 23: 368-374, 1975

25. Lisk, R. D., J. Zeiss and L. A. Ciaccio. The influence of olfac tion on sexual behavior in the male golden hamster 1 Mesocricetus atratus). J. exp. Zool. 181: 69-78, 1972

26. Macrides, F., A. Bartke, F. Fernandez and W. D Angelo. Effects of exposure to vaginal odor and receptive females on plasma testosterone in the male hamster. Neuroendocrinolog! 15: $355-364,1974$

27. Macrides, F., P. A. Johnson and S. P. Schneider. Responses of the male golden hamster to vaginal secretion and dimethyl disulfide: Attraction versus sexual behavior. Behat. Biol. 20: 377-386, 1977 .

28. Meredith, M. Efferent control of stimulus access to the vomeronasal organ (VNO). Neurosci. Abstr. 2: 159, 1976.

29. Muller-Schwarze, D. and M. M. Mozell (Editors). Chemical Signals in Vertebrates. New York: Plenum Press, 1976.

30. Murphy, M. R. Effects of female hamster vaginal discharge on the behavior of male hamsters. Behai. Biol. 9: 367-375, 1973.

31. Murphy, M. R. Olfactory stimulation and olfactory bulb removal: Effects on territorial aggression in male syrian golden hamsters. Bruin Res. 113: 95-110, 1976

32. Murphy, M. R. Olfactory impairment, olfactory bulb removal, and mammalian reproduction. In: Mammalian Olfaction, $R_{e}$ productive Processes, and Behurior, edited by R. L. Doty. New York: Academic Press, 1976, pp. 95-117.

33. Negus, V. Comparative Anatomy and Phsiology of the Nose and Parallasal Sinuses. Edinburgh: Livingstone. 1958, pp 103-129.

34. Nyby, J., C. J. Wysocki, G. Whitney and G. Dizinno. Pheromonal regulation of male mouse ultrasonic courtship (Mus mus(ullss). Anim. Behav. 25: 333-341, 1977

35. Powers, J. B. and S. S. Winans. Sexual behavior in peripherally anosmic male hamsters. Phwsiol. Behav. 10: 361-368, 1973

36. Powers, J. B. and S. S. Winans. Vomeronasal organ: Critical role in mediating sexual behavior of the male hamster. Science 187: $961-963,1975$

37. Regnier, F. E. and M. Goodwin. On the chemical and environmental modulation of pheromone release from vertebrate scen marks. In: Chemical Signals in Vertehrates, edited by D Muller-Schwarze and M. M. Mozell. New York: Plenum Press 1976, pp. $115-133$

38. Scalia, F. and S. S. Winans. The differential projections of the olfactory bulb and accessory olfactory bulb in mammals. $J$ comp. Newol. 161: 31-56, 1975.

39. Singer, A. G., W. C. Agosta, R. J. O'Connell, C. Pfaffman, D V. Bowen and F. H. Field. Dimethyl disulfide: An attractant pheromone in hamster vaginal secretion. Scitence 191: 948-950 1976.

40. Tucker. D. Nonolfactory responses from the nasal cavity Jacobson's organ and the trigeminal system. In: Olfaction Handhook of Sensory Physiology, vol. 4, edited by L. M. Bei dler. New York: Springer-Verlag, 1971, pp. 151-181.

41. Verberne, $G$. Beobachtungen und versuche uber das Flehmen Katzenartiger Raubtiere. Z. Titerpsyc/hol. 27: 807-827, 1970.

42. Winans. S. S. and J. B. Powers. Olfactory and vomeronasa deafferentation of male hamsters: Histological and behavioral analyses. Brain Res. 126: 325-344. 1977. 